\title{
Assessment of perioperative minute ventilation in obese versus non-obese patients with a non-invasive respiratory volume monitor
}

\author{
Jaideep H. Mehta ${ }^{1}$, Davide Cattano ${ }^{1}$, Jordan B. Brayanov ${ }^{2 *}$ and Edward E. George ${ }^{3}$
}

\begin{abstract}
Background: Monitoring the adequacy of spontaneous breathing is a major patient safety concern in the postoperative setting. Monitoring is particularly important for obese patients, who are at a higher risk for post-surgical respiratory complications and often have increased metabolic demand due to excess weight. Here we used a novel, noninvasive Respiratory Volume Monitor (RVM) to monitor ventilation in both obese and non-obese orthopedic patients throughout their perioperative course, in order to develop better monitoring strategies.

Methods: We collected respiratory data from 62 orthopedic patients undergoing elective joint replacement surgery under general anesthesia using a bio-impedance based RVM with an electrode PadSet placed on the thorax. Patients were stratified into obese $(\mathrm{BMI} \geq 30)$ and non-obese cohorts and minute ventilation (MV) at various perioperative time points was compared against each patient's predicted minute ventilation ( $\left(\mathrm{MV}_{\mathrm{PRED}}\right)$ based on ideal body weight (IBW) and body surface area (BSA). The distributions of MV measurements were also compared across obese and non-obese cohorts.

Results: Obese patients had higher MV than the non-obese patients before, during, and after surgery. Measured MV of obese patients was significantly higher than their MV PRED from IBW formulas, with BSA-based MV PRED being a closer estimate. Obese patients also had greater variability in MV post-operatively when treated with standard opioid dosing.

Conclusions: Our study demonstrated that obese patients have greater variability in ventilation post-operatively when treated with standard opioid doses, and despite overall higher ventilation, many of them are still at risk for

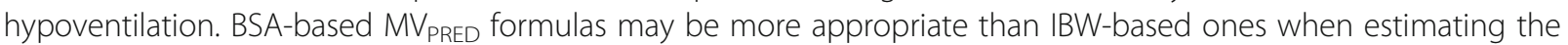
respiratory demand of obese patients. The RVM allows for the continuous and non-invasive assessment of respiratory function in both obese and non-obese patients.
\end{abstract}

Keywords: Respiratory monitoring, Respiratory requirements, Minute ventilation, Perioperative safety, Opioids, Obesity

\footnotetext{
* Correspondence: Jordan.Brayanov@respiratorymotion.com

${ }^{2}$ Respiratory Motion Inc., 411 Waverley Oaks Rd \#150, Waltham, MA 02452,

USA

Full list of author information is available at the end of the article
}

(c) The Author(s). 2017 Open Access This article is distributed under the terms of the Creative Commons Attribution 4.0 International License (http://creativecommons.org/licenses/by/4.0/, which permits unrestricted use, distribution, and reproduction in any medium, provided you give appropriate credit to the original author(s) and the source, provide a link to the Creative Commons license, and indicate if changes were made. The Creative Commons Public Domain Dedication waiver (http://creativecommons.org/publicdomain/zero/1.0/) applies to the data made available in this article, unless otherwise stated. 


\section{Background}

Adequate respiratory monitoring is necessary for patients undergoing surgery, and is especially crucial in the postoperative period where the patient's airway is not secured and staff presence is intermittent. Several factors can lead to respiratory compromise and respiratory depression, with the use of sedatives and opioids being a prominent example. Obstructive breathing patterns, often unappreciated preoperatively, can be initiated or exacerbated by anesthetics and opioids and trigger postoperative apnea (POA). It has been repeatedly shown that patient controlled analgesia (PCA), which was thought to be a safe alternative to staff-administered analgesia, has not eliminated opioid induced respiratory depression (OIRD) which remains one of the most dangerous adverse postoperative outcomes $[1,2]$.

Postoperative apnea (POA), unlike other forms of apnea, is often a combination of both obstructive and central apnea [3]. The effect of opioids on respiratory drive results from both direct depressant action on respiratory neural activity as well as from the reduction in pharyngeal muscle tone. Obese patients account for up to $58 \%$ of adult obstructive sleep apnea (OSA) cases and a $10 \%$ increase in body mass leads to a six-fold increase in the risk of developing moderate to severe OSA [4]. Obstructive respiratory patterns, postoperative pulmonary atelectasis, [5] reduced functional residual capacity, reduced vital capacity, and increased metabolic (and respiratory) demand all lead to increased perioperative risk in obese patients [5-9]. With these risk factors in mind, respiratory monitoring and timely identification of respiratory insufficiency in the perioperative period is paramount for obese patients.

Indirect respiratory monitoring like oxygen saturation $\left(\mathrm{SpO}_{2}\right)$ and capnography $\left(\mathrm{EtCO}_{2}\right)$ are unable to identify early signs of respiratory compromise and can delay interventions [10]. Moreover, both $\mathrm{SpO}_{2}$ and $\mathrm{EtCO}_{2}$ monitoring systems are plagued by false alarms in the post-operative setting $[11,12]$. To address these issues, a non-invasive respiratory volume monitor (RVM) providing direct, realtime measures of minute ventilation (MV), tidal volume $(\mathrm{TV})$ and respiratory rate (RR) in non-intubated patients has been developed.

Previous research shows that the RVM can reliably provide accurate real-time continuous respiratory volume measurements when compared against a monitoring spirometer or a ventilator, with approximately 90\% accuracy for MV and TV, and 98\% accuracy for RR [13, 14]. The RVM applies a small amount of high-frequency electrical current to the patient's thorax and monitors changes in the electrical signal using three EKG-like electrodes placed at the sternal notch, xiphoid and right mid-axillary line. The RVM uses the difference in conductivity between air and tissue to calculate the amount of air moving in and out of the lung in real time and displays an easily interpretable breath-by-breath waveform as well as 30-second averages for MV, TV and RR.

This RVM has also been shown to help identify "true" desaturation events, which can help reduce the rate of false SpO2 alarms due to patient movement and other variables. This is particularly useful in more mobile patients recovering from surgery [15]. In addition, changes in MV have been shown to reflect the patient's actual respiratory status quicker and with better fidelity than conventional EtCO2 monitoring [16, 17].

In this study we used the RVM to monitor respiratory status in obese and non-obese orthopedic patients and compare ventilation between these two patient categories throughout their perioperative course. We also compared measured MV to known standards of predicted $\mathrm{MV}$ in an attempt to better understand the differences in metabolic (respiratory) demand amongst the obese patients.

\section{Methods}

This study was conducted at the Massachusetts General Hospital (MGH) and the Partners Institutional Review Board approved its design (2011P002898, Boston, MA). All patients provided written informed consent prior to enrollment. Inclusion criteria were English-speaking men and women aged 18 to 99 years undergoing elective joint replacement surgery. There were no specific exclusion criteria. Here, we specifically selected patients undergoing surgery under general anesthesia so that we could have data as to the ventilator settings used during surgery.

We used a bio-impedance based RVM (ExSpiron 1Xi, Respiratory Motion, Waltham, MA) to collect digital respiratory data via an electrode PadSet placed on the thorax as previously described (See Fig. 1 in Voscopoulos et al., 2014 ${ }^{3}$ ). We also collected pertinent demographic, physiologic, and clinical data. The RVM and physiologic data were collected beginning in preoperative holding, throughout the surgical procedure, and ending post-operatively following sign-out from the post-anesthesia care unit (PACU). Note that, all clinical staff involved in the patient care (surgery, anesthesia, PACU staff, etc....) were blinded to the RVM measurements. As such, the RVM was not used to inform or alter patient care, and was only used to collect respiratory data for research.

During post-hoc analysis of the data, patients were stratified according to obesity status (BMI $\geq 30 \mathrm{~kg} / \mathrm{m}^{2}$ ) and observed MV was compared against the patient's predicted $\mathrm{MV}$ ( $\left.\mathrm{MV}_{\mathrm{PRED}}\right)$ based on both ideal body weight (IBW) and body surface area (BSA) formulas [18, 19]. Ventilator MV (Apollo, Draeger, Telford, PA) was also collected during general anesthesia and was assumed to be "adequate" as it was adjusted to maintain appropriate 


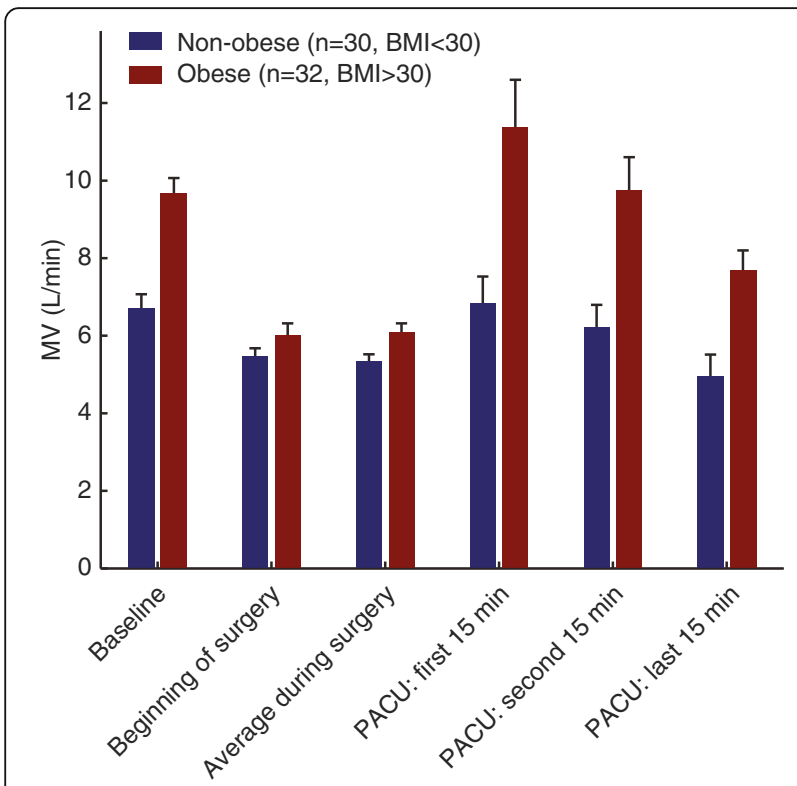

Fig. 1 Comparison of MV measurements in obese (Red, BMI $\geq 30$ ) and non-obese (Blue, BMl<30) patients at various points throughout their perioperative course. The measured MV in the obese population was significantly higher at every time point throughout the course: preoperatively (Baseline, $9.7 \pm 0.4$ vs. $6.7 \pm 0.3 \mathrm{~L} / \mathrm{min}$ ), beginning of surgery $(6.0 \pm 0.3$ vs. $5.5 \pm 0.2 \mathrm{~L} / \mathrm{min})$, during surgery (6.1 \pm 0.2 vs. $5.3 \pm 0.2)$, on arrival at the PACU (first $15 \mathrm{~min}: 11.4 \pm 1.2 \mathrm{vs} .6 .8 \pm 0.7 \mathrm{~L} / \mathrm{min}$ and second $15 \mathrm{~min}: 9.7 \pm 0.8$ vs. $6.2 \pm 0.6 \mathrm{~L} / \mathrm{min}$ ) and at discharge (last $15 \mathrm{~min}: 7.7 \pm 0.5$ vs. $5.0 \pm 0.6 \mathrm{~L} / \mathrm{min})$. These results are incongruent with IBW-based predicted MV (MVPRED) which were very similar $(6.2 \pm 0.2 \mathrm{vs}$. $6.1 \pm 0.2 \mathrm{~L} / \mathrm{min}, p=0.37$ ) and more closely aligned with BSA-based $M_{\text {PRED }}(7.8 \pm 0.2$ vs. $6.9 \pm 0.2 \mathrm{~L} / \mathrm{min}, p=0.0002)$

$\mathrm{EtCO}_{2}$ during surgery. The number of opioid doses (either $1 \mathrm{mg}$ morphine or $0.2 \mathrm{mg}$ hydromorphone) as well as the total morphine equivalent of opioids received in the PACU were compared across the two cohorts. We used a conversion factor of $0.16 \mathrm{mg}$ hydromorphone $=1 \mathrm{mg}$ morphine equivalent (MME) and $1 \mathrm{mg}$ of morphine $=1 \mathrm{MME}$ [20].

The main goal of this study was to assess and report on any differences in respiratory volumes between obese and non-obese patients. The secondary goals of the study assessed whether (a) predicted MV formulas based on IBW or BSA would more adequately capture the actual MV observed in the 2 patient cohorts and (b) differences in opioid use in the PACU could explain any potential differences in MV.

Unpaired two-tailed t-tests were used to compare the MV measurements and opioid dosing between the two patient subgroups (non-obese vs. obese) at several time points of the study. Specifically, comparisons were made pre-operatively (Baseline), at the beginning of surgery, during surgery, the first and second 15 min upon arrival at the PACU, and the last $15 \mathrm{~min}$ prior to discharge. Two-sample F-tests for equal variance were used to compare distributions of $\mathrm{MV}$ at these same time points.
Since we had collected multiple [repeated] measurements from each patient in this study, we used a more conservative $\mathrm{p}<0.01$ cutoff to evaluate the significance of our findings in order to account for any effects of auto-correlation amongst within-patient measurements. All analyses were performed in Matlab 2012b.

\section{Results}

In this study we enrolled 62 orthopedic patients (Age: $65.4 \pm 12.0$ years, BMI: $30.8 \pm 6.0 \mathrm{~kg} / \mathrm{m}^{2}$ ), 32 of them were classified as obese and 30 as non-obese. Both the obese and non-obese cohorts had similar $M V_{\text {PRED }}$ based on ideal body weight (IBW): Obese: $6.2 \pm 0.2$; Non-Obese: $6.1 \pm$ $0.2 \mathrm{~L} / \mathrm{min}, \mathrm{p}>0.3$, since IBW-based $\mathrm{MV}_{\text {PRED }}$ formulas are entirely based on patient's height and sex and not their weight. However, obese patients were managed during surgery at a significantly higher MV while intubated (6.1 \pm 0.2 vs. $5.3 \pm 0.2 \mathrm{~L} / \mathrm{min}, p=0.0068)$, suggesting that IBWbased $M V_{\text {PRED }}$ may not adequately capture the increase in metabolic demand in obese patients.

If instead we use $M V_{\text {PRED }}$ calculated based on each patient's Body Surface Area (BSA), we see that $M V_{\text {PRED }}$ for the obese population is significantly higher than the non-obese $(7.8 \pm 0.2$ vs. $6.9 \pm 0.2 \mathrm{~L} / \mathrm{min}, \mathrm{p}=0.0002)$. Using BSA instead of IBW increased $M V_{\text {PRED }}$ in the obese by more than $25 \%$, while the increase in the nonobese was just $11 \%$, suggesting that IBW or BSA -based $M V_{\text {PRED }}$ are similar in non-obese patients, but differ substantially in the obese.

During periods of spontaneous breathing perioperatively, the differences in MV between the two cohorts were even more significant, as summarized in Fig. 1. Obese patients systematically had higher MV than nonobese patients pre-operatively $(9.7 \pm 0.4$ vs. $6.7 \pm 0.3 \mathrm{~L} /$ min, $\mathrm{p}=0.0000003)$, at PACU arrival $(11.4 \pm 1.2 ; 6.8 \pm$ $0.7 \mathrm{~L} / \mathrm{min}, \mathrm{p}=0.0014)$, and at PACU discharge $(7.7 \pm 0.5$; $5.0 \pm 0.6 \mathrm{~L} / \mathrm{min}, p=0.0004)$. All of these differences were significant at a level of $p<0.01$. Note that, 11 of the 62 patients here $(17.7 \%)$ had a previous diagnosis of OSA, 9 of these patients with known OSA were obese $(28.1 \%$ of the obese cohort) and 2 were non-obese $(6.7 \%$ of the non-obese cohort). None of these patients with known OSA received any specific therapy or treatment related to their OSA in the PACU.

However, while obese patients had generally higher MV than the non-obese, a substantial fraction of obese patients experienced respiratory depression in the PACU (see Fig. 2). In fact, $10 \%$ of the obese patients spent more than $1 / 3$ of their last $30 \mathrm{~min}$ in the PACU with un-safe $\mathrm{MV}\left(\mathrm{MV}<40 \% \mathrm{MV}_{\mathrm{PRED}}\right)$ [3]. In the non-obese population, the fraction of patients with Low MV for $>1 / 3$ of the last $30 \mathrm{~min}$ was substantially higher (37\%), which could be due to the similar dosing of opioids in both groups despite differences in patient body mass. As Fig. 3 


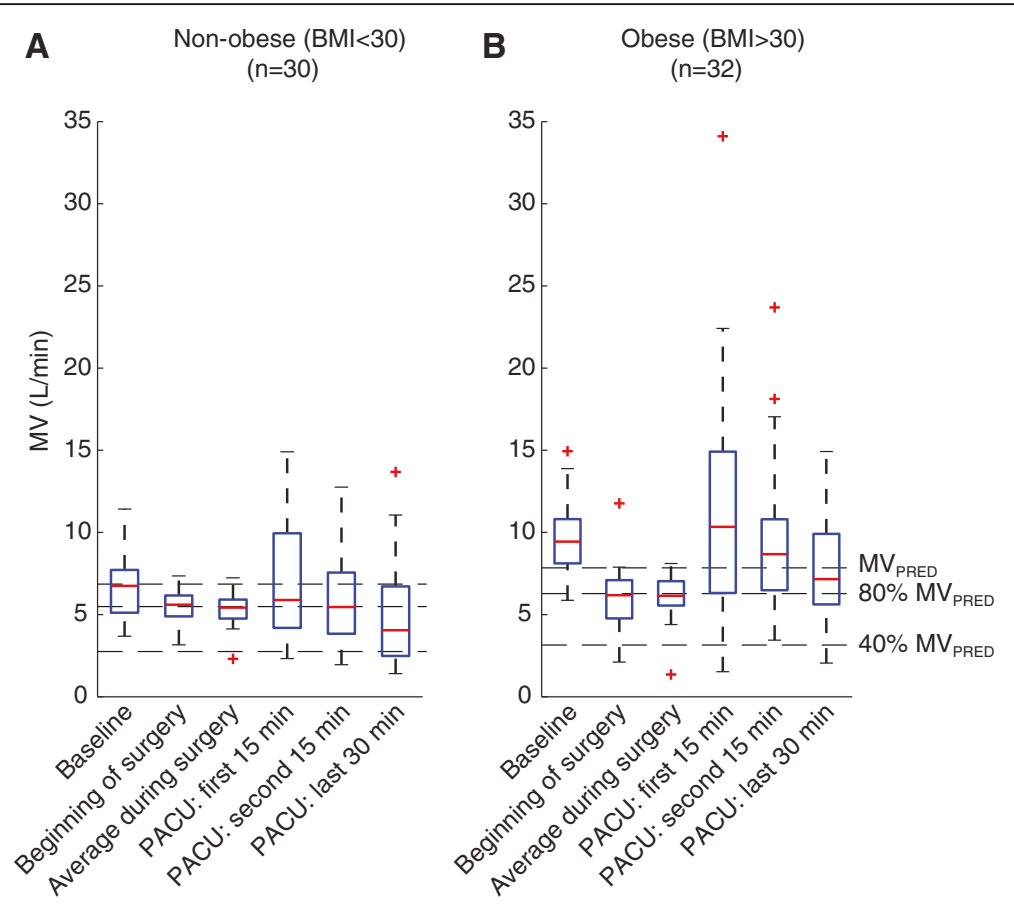

Fig. 2 Patient-to-patient variability in minute ventilation (MV) in non-obese and obese orthopedic patients at different time-points in the perioperative course. Boxplots show MV mean and variance in (a) non-obese and (b) obese orthopedic patient cohorts at seven time-points pre-operatively, intraoperatively, and post-operatively. Dashed lines depict BSA-based MV PRED, as well as at-risk (80\% MV PRED) and un-safe (40\% MV PRED) ventilation thresholds. Post-operative MV variance is significantly greater in obese patients than in non-obese patients $(p<0.01)$, particularly in the PACU, and whereas the average MV at PACU discharge in the obese patients is significantly higher than in the non-obese patients, $10 \%$ of the obese patients spent $>1 / 3$ of the last half-hour in the PACU with un-safe MV

shows, there was no significant difference in the number of PCA opioid doses administered to the obese vs. non-obese group ( $3.7 \pm 0.7$ vs. $4.7 \pm 1.2$ doses, $p>0.05$, Fig. $3 a)$ or the total morphine equivalence of opioids administered in the PACU ( $4.7 \pm 1.0$ vs. $6.0 \pm 1.6 \mathrm{MME}, p>0.05$, Fig. $3 \mathrm{~b})$.

\section{Discussion}

We noted significant differences in MV across the two cohorts, with the obese patients systematically sustaining higher MV. This is at odds with the predicted MV $\left(M V_{\text {PRED }}\right)$ based on IBW and much more consistent with $M V_{\text {PRED }}$ based on BSA. Additionally, MV measurements for obese patients pre- and post-operatively (while spontaneously breathing and not intubated) were often significantly higher than while intubated and on the ventilator. Yet a significant fraction of the obese patients suffered transient respiratory depression (episodes of $\mathrm{MV}<40 \%$ $\left.M V_{\text {PRED }}\right)$ while treated with standard-dose opioids.

This is the first study to compare the perioperative respiratory demand (in the form of minute ventilation) between obese and non-obese patients, which only became possible since the development and clinical implementation of a non-invasive respiratory volume monitor. According to a recent study, [16] $\mathrm{EtCO}_{2}$ measurements could not adequately characterize rapid

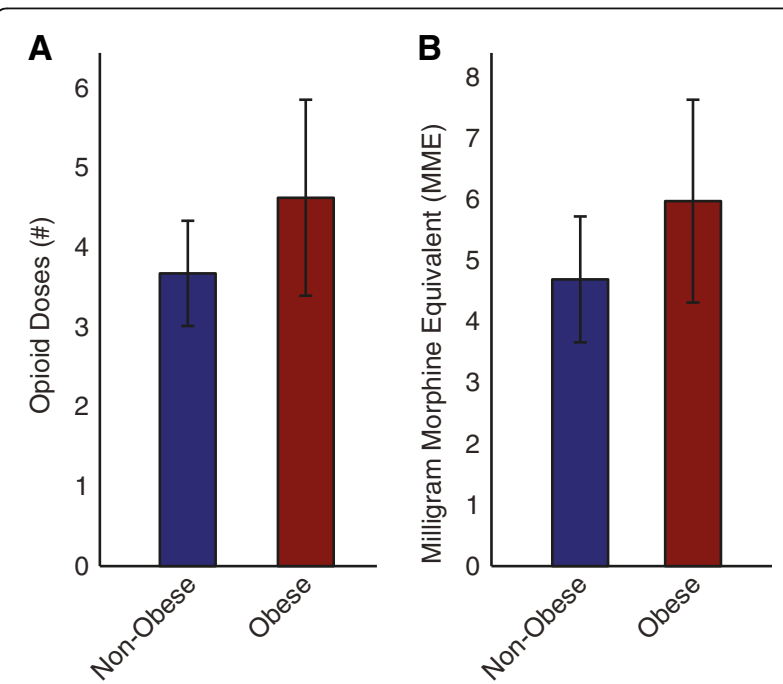

Fig. 3 Opioid usage in the PACU in non-obese (blue) and obese (red) orthopedic patients. (a) Average number of opioid doses administered over the course of a PACU stay in the obese and non-obese orthopedic patient cohort, respectively. (b) Average total morphine equivalent dose of opioids administered in the PACU. Error bars represent the standard error of the mean (SEM). There is no significant difference observed in PACU opioid dosage when patients are stratified for obesity $(p>0.05$ for both comparisons) 
changes in ventilation either with an in-line sensor, or with an oral/nasal cannula. Several previous studies have shown that the RVM is a more accurate and reliable monitoring option than alternative monitoring technologies such as capnography $[13,14]$. This is particularly important in procedural sedation and non-operating room anesthesia, where $\mathrm{EtCO} 2$ and oxygen saturation are usually the only available monitoring modalities for respiratory insufficiency, yet are often unreliable or delayed [17].

Since the accuracy of the RVM measurements has been previously demonstrated, the RVM now can provide useful clinical data implications to improve assessment and treatment in specific patient populations The objective of this work was to assess respiratory parameters in non-intubated obese versus non-obese patients, which had not previously been possible. Although it will be important to extend this work to more specific evaluations, here we did not analyze the effects of intraoperative opioids, paralytics, and anesthetic agents and we did not stratify patients based on their other comorbidities or confounding factors. A limitation to the study is that uniformly recorded pain scores were not available from the PACU. While potentially interesting, EtCO2 measurements were not part of standard clinical care and were not obtained during this study. Additional work is needed to help extend these preliminary findings to optimize clinical practice.

A distinct difference in the patient-to-patient respiratory variability became evident between the obese and non-obese cohorts (Fig. 2). Pre-operatively both patient cohorts exhibited similar patient-to-patient variability in MV (i.e. the distribution of MV measurements across patients had similar variance), whereas in the postoperative period the patient-to-patient variability in the obese cohort was significantly larger. This suggests that some obese patients, despite generally having higher minute ventilation, experience episodes of hypoventilation in the PACU associated with unpredictable swings in MV. By monitoring changes in both respiratory rate and tidal volume, individualized treatment plans could be implemented for obese patients.

Specifically in the obese population, Schumann et al. [21] previously suggested that traditional IBW-based MV nomograms may be suboptimal in the defining Predicted MV. Our findings support that conclusion and suggest the preferential use of BSA-based formulas. In this study, the PACU opioid dosage was not significantly different between the obese and non-obese patients, suggesting the differences observed in MV across the two groups cannot be attributed to a greater opioid dosage.

This study demonstrated the utility of the RVM in defining baseline respiratory characteristics in obese vs. non-obese patients. Importantly, real-time respiratory monitoring could help define each individual patient's respiratory demand and enable individualized care. This could enable deeper understanding of the underlying physiology and help prevent respiratory depression and improve patient safety.

\section{Conclusions}

Our study demonstrated that obese patients have greater variability in ventilation post-operatively when treated with standard opioid doses, and despite overall higher ventilation, many of them are still at risk for hypoventilation. The conventional IBW-based $\mathrm{MV}_{\text {PRED }}$ formulas may not be accurate predictors of expected respiratory performance in obese patients. Instead, BSA-based MV PRED formulas appear to capture the baseline function and real-time changes in metabolic demand in the obese population more accurately. In addition, the use of the RVM may allow for the continuous and non-invasive assessment of respiratory function, provide more clinically useful data than either $\mathrm{EtCO} 2$ or $\mathrm{SpO} 2$, and assist in the adjustment of treatment to individualize care in both obese and non-obese patients.

\section{Abbreviations \\ BSA: Body surface area; $\mathrm{EtCO}_{2}$ : End-tidal carbon dioxide; IBW: Ideal body weight; MME: Morphine-milligram equivalent; MV: Minute ventilation; MV PRED: Predicted minute ventilation; PACU: Post-anesthesia care unit; PCA: Patient-controlled analgesia; RR: Respiratory rate; RVM: Respiratory volume monitor; TV: Tidal volume.}

\section{Acknowledgements}

Not applicable.

\section{Funding}

This study was supported by Respiratory Motion Inc who loaned RVM monitors to the Massachusetts General Hospital and provided the disposables used for this study.

\section{Availability of data and materials}

The datasets during and/or analysed during the current study are available from the corresponding author on reasonable request.

\section{Authors' contributions}

JM assisted with data analysis and interpretation and drafting of the manuscript. DC assisted with the interpretation of the data, the positioning of our findings in clinically-relevant context, and drafting of the manuscript. JB assisted with study design, data collection and analysis, and drafting of the manuscript. EG assisted with study design, data collection, and drafting of the manuscript. All authors read and approved the final manuscript.

\section{Competing interests}

JM owns stock in Respiratory Motion Inc. JB was an employee of Respiratory Motion Inc. at the time this article was completed.

Consent for publication

Not applicable, no individual data was reported.

Ethics approval and consent to participate

The study was approved by the Partners Institutional Review Board (2011P002898), and all subjects provided written informed consent prior to enrolment.

\section{Publisher's Note}

Springer Nature remains neutral with regard to jurisdictional claims in published maps and institutional affiliations. 


\section{Author details}

University of Texas Medical School at Houston, 6431 Fannin Street, Houston, TX 77030, USA. ${ }^{2}$ Respiratory Motion Inc., 411 Waverley Oaks Rd \#150, Waltham, MA 02452, USA. ${ }^{3}$ Massachusetts General Hospital, Harvard Medical School, 55 Fruit St, Boston, MA 02114, USA.

Received: 2 September 2016 Accepted: 21 April 2017

Published online: 26 April 2017

\section{References}

1. Willens JS, Jungquist CR, Junquist CR, Cohen A, Polomano R. ASPMN survey-nurses' practice patterns related to monitoring and preventing respiratory depression. Pain Manag Nurs. 2013;14:60-5.

2. Pasero C. Opioid-induced sedation and respiratory depression: evidencebased monitoring guidelines. J Perianesth Nurs. 2012;27:208-11.

3. Voscopoulos CJ, MacNabb CM, Freeman J, Galvagno SM, Ladd D, George E. Continuous noninvasive respiratory volume monitoring for the identification of patients at risk for opioid-induced respiratory depression and obstructive breathing patterns. J Trauma Acute Care Surg. 2014;77:S208-15.

4. Young T, Peppard PE, Taheri S. Excess weight and sleep-disordered breathing. J Appl Physiol. 2005;99:1592-9.

5. Eichenberger A-S, Proietti S, Wicky S, Frascarolo P, Suter M, Spahn DR, et al. Morbid obesity and postoperative pulmonary atelectasis: an underestimated problem. Anesth Analg. 2002;95:1788-92. table of contents.

6. Fung E, Cave D, Witmans M, Gan K, El-Hakim H. Postoperative respiratory complications and recovery in obese children following adenotonsillectomy for sleep-disordered breathing: a case-control study. Otolaryngol Head Neck Surg. 2010;142:898-905.

7. Mendonça J, Pereira H, Xará D, Santos A, Abelha FJ. Obese patients: respiratory complications in the post-anesthesia care unit. Rev Port Pneumol. 2014;20:12-9.

8. Pasulka PS, Bistrian BR, Benotti PN, Blackburn GL. The risks of surgery in obese patients. Ann Intern Med. 1986;104:540-6.

9. Hans GA, Lauwick S, Kaba A, Brichant J-F, Joris JL. Postoperative respiratory problems in morbidly obese patients. Acta Anaesthesiol Belg. 2009;60:169-75

10. Lee LA, Caplan RA, Stephens LS, Posner KL, Terman GW, Voepel-Lewis T, et al. Postoperative opioid-induced respiratory depression: a closed claims analysis. Anesthesiology. 2015:122:659-65.

11. Drew BJ, Harris P, Zègre-Hemsey JK, Mammone T, Schindler D, Salas-Boni R, et al. Insights into the problem of alarm fatigue with physiologic monitor devices: a comprehensive observational study of consecutive intensive care unit patients. PLoS One. 2014;9:e110274.

12. Petterson MT, Begnoche VL, Graybeal JM. The effect of motion on pulse oximetry and its clinical significance. Anesth Analg. 2007;105:S78-84

13. Voscopoulos C, Brayanov J, Ladd D, Lalli M, Panasyuk A, Freeman J. Special article: evaluation of a novel noninvasive respiration monitor providing continuous measurement of minute ventilation in ambulatory subjects in a variety of clinical scenarios. Anesth Analg. 2013;117:91-100.

14. Voscopoulos CJ, MacNabb CM, Brayanov J, Qin L, Freeman J, Mullen GJ, et al. The evaluation of a non-invasive respiratory volume monitor in surgical patients undergoing elective surgery with general anesthesia. J Clin Monit Comput. 2015;29:223-30

15. Galvagno S, Duke P, Eversole D, George E. Evaluation of Respiratory Volume Monitoring (RVM) to Detect Respiratory Compromise in Advance of Pulse Oximetry and Help Eliminate False Desaturation Alarms. J Trauma Acute Care Surg. 2016;81(5 Suppl 2 Proceedings of the 2015 Military Health System Research Symposium):S162-S170.

16. Williams GW, George CA, Harvey BC, Freeman JE. A Comparison of Measurements of Change in Respiratory Status in Spontaneously Breathing Volunteers by the ExSpiron Noninvasive Respiratory Volume Monitor Versus the Capnostream Capnometer. Anesth Analg. 2017;124(1):120-6.

17. Ebert TJ, Middleton AH, Makhija N. Ventilation monitoring during moderate sedation in Gl patients. J Clin Monit Comput. 2017;31(1):53-7.

18. Devine B. Gentamicin therapy. Drug Intell Clin Pharm. 1974:8:650-5.

19. Du Bois D, Du Bois EF. A formula to estimate the approximate surface area if height and weight be known. 1916. Nutrition. 1989;5:303.

20. Gammaitoni AR, Fine P, Alvarez N, McPherson ML, Bergmark S. Clinical application of opioid equianalgesic data. Clin J Pain. 2003;19:286-97.

21. Schumann R, Kwater AP, Bonney I, Ladd D, Kim J, Gupta A, et al. Respiratory volume monitoring in an obese surgical population and the prediction of postoperative respiratory depression by the STOP-bang OSA risk score. J Clin Anesth. 2016;34:295-301.

\section{Submit your next manuscript to BioMed Central and we will help you at every step:}

- We accept pre-submission inquiries

- Our selector tool helps you to find the most relevant journal

- We provide round the clock customer support

- Convenient online submission

- Thorough peer review

- Inclusion in PubMed and all major indexing services

- Maximum visibility for your research

Submit your manuscript at www.biomedcentral.com/submit
C) Biomed Central 